\title{
Addendum modification of spur gears with equalized efficiency at the points where the meshing stars and ends
}

\author{
Antal Tiberiu Alexandru \\ The Technical University from Cluj-Napoca, C. Daicoviciu no. 15, 400020, Cluj-Napoca, Romania, \\ E-mail: Tiberiu.Alexandru.Antal@mep.utcluj.ro
}

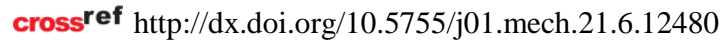

\section{Introduction}

When determining the geometric dimensions of cylindrical spur gears, most often, specific addendum modifications are used. Their purpose is to obtain a well defined distance between the axes at the same time ensuring the correct meshing of teeth flanks over a longer time. The $x_{1}$ and $x_{2}$ addendum modification coefficients have influence on tooth shape and tooth thickness. Different assumptions or criteria can be used to determine their values as shown in [1] - [7]. Balancing or equalization of the sliding velocity [1], specific sliding [1,2] or power lost by friction [3] are used to influence friction losses to obtain better efficiency, lower operating temperature, noise and wearing to increase the life of the gears. Instead of equalizing the power losses the paper presents a new method to establish the specific addendum modification coefficients using the equalization of the efficiencies, at the $\mathrm{A}$ and $\mathrm{E}$ points, where the meshing begins and ends (see Fig. 1). Compared to [2] this method is considering friction coefficients with distinct values between the teeth flanks at beginning and the end of the contact allowing to study the gear efficiency in a general case. Compared to [3] the method is dealing directly with the efficiency - the most important objective in the field of transmissions - and allows the study of the balanced gear efficiency for various friction coefficients and addendum modifications.

\section{Efficiencies at the beginning and end of the teeth meshing}

The efficiency of the teeth meshing is determined using the following general relation:

$$
\eta=\frac{T_{2} \omega_{2}}{T_{1} \omega_{1}}=\frac{T_{2} z_{1}}{T_{1} z_{2}}
$$

where $T_{1}$ is the torque of the driving forces; $T_{2}$ the torque of the useful resistance forces; $\omega_{1}$ the angular velocity of the driving wheel; $\omega_{2}$ the angular velocity of the driven wheel; $z_{1}$ the number of teeth of the driving wheel; $z_{2}$ the number of teeth of the driven wheel.

Considering Fig. 1 the torque at the beginning of the teeth meshing, in point $\mathrm{A}$, can be written with the following expressions:

$$
T_{1}=F_{n A}\left[r_{b 1}\left(1-\mu_{A} \tan \left(\alpha_{w}\right)\right)+\mu_{A} e_{A}\right]
$$

and

$$
T_{2}=F_{n A}\left[r_{b 2}\left(1-\mu_{A} \tan \left(\alpha_{w}\right)\right)-\mu_{A} e_{A}\right] .
$$

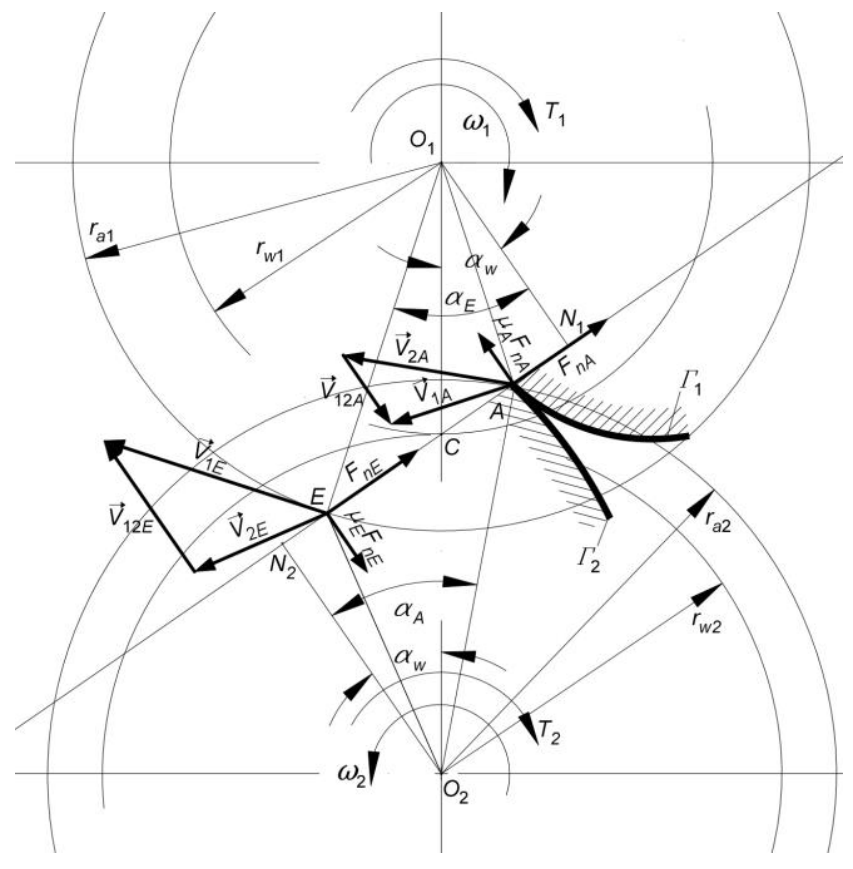

Fig. 1 Meshing of involute spur gears

where $F_{n A}$ is the normal force in the A meshing point, $r_{b 1}$ and $r_{b 2}$ the base circles radii of the meshing gears with involute profiles $\left(r_{b 2} z_{1}=r_{b 1} z_{2}\right), \mu_{A}$ the friction coefficient between the teeth flanks, in the A point, where the meshing begins, $\alpha_{w}$ the meshing angle, $e_{A}$ the AC segment length from Fig. 1. From (2) and (3) the meshing efficiency in the A point becomes:

$$
\eta_{A}=\frac{z_{1}\left(1-\mu_{A} \tan \left(\alpha_{A}\right)\right)}{z_{1}\left(1-\mu_{A} \tan \left(\alpha_{A}\right)\right)+z_{2} \mu_{A}\left(\tan \left(\alpha_{A}\right)-\tan \left(\alpha_{w}\right)\right)} .
$$

Using the same methodology the torque is determined in the E point, where the meshing ends, thus:

$$
T_{1}=F_{n E}\left[r_{b 1}\left(1+\mu_{E} \tan \left(\alpha_{w}\right)\right)+\mu_{E} e_{E}\right]
$$

and

$$
T_{2}=F_{n E}\left[r_{b 2}\left(1+\mu_{E} \tan \left(\alpha_{w}\right)\right)-\mu_{E} e_{E}\right],
$$

where $F_{n E}$ is the normal force in the E meshing point, $\mu_{E}$ the friction coefficient between the teeth flanks, in the $\mathrm{E}$ point, $e_{E}$ the EC segment length from Fig. 1.

From (5) and (6) the meshing efficiency in the E point becomes:

$\eta_{E}=\frac{z_{2}\left(1+\mu_{E} \tan \left(\alpha_{w}\right)\right)-z_{1} \mu_{E}\left(\tan \left(\alpha_{E}\right)-\tan \left(\alpha_{w}\right)\right)}{z_{2}\left(1+\mu_{E} \tan \left(\alpha_{E}\right)\right)}$. 
In order to check the possibility of making equal the (4) and (6) expressions we will compute and plot these surfaces depending on $x_{1}$ and $x_{2}$ addendum modifications.

\section{Computation and graphical representation of the efficiency}

The numerical results for the (4) and (7) expressions are obtained using the following known formulae [6] from the spur gear geometry:

$$
\begin{aligned}
& \cos \left(\alpha_{A}\right)=\frac{r_{2}}{r_{a 2}} \cos \left(\alpha_{0}\right) ; \\
& \cos \left(\alpha_{E}\right)=\frac{r_{1}}{r_{a 1}} \cos \left(\alpha_{0}\right) ; \\
& r_{1}=\frac{m z_{1}}{2} ; \\
& r_{2}=\frac{m z_{2}}{2} ; \\
& r_{a 1}=\frac{m}{2}\left(z_{1}+h_{a}^{*}+2 x_{1}-2 k\right) ; \\
& r_{a 2}=\frac{m}{2}\left(z_{2}+h_{a}^{*}+2 x_{2}-2 k\right) ; \\
& k=x_{1}+x_{2}-y ; \\
& y=\frac{z_{1}+z_{2}}{2}\left(\frac{\cos \left(\alpha_{0}\right)}{\cos \left(\alpha_{w}\right)}-1\right),
\end{aligned}
$$

where $\alpha_{w}$ is computed from the following equation:

$$
x_{1}+x_{2}=\left[\tan \left(\alpha_{w}\right)-\alpha_{w}-\left(\tan \left(\alpha_{0}\right)-\alpha_{0}\right)\right] \frac{z_{1}+z_{2}}{2 \tan \left(\alpha_{0}\right)} .
$$

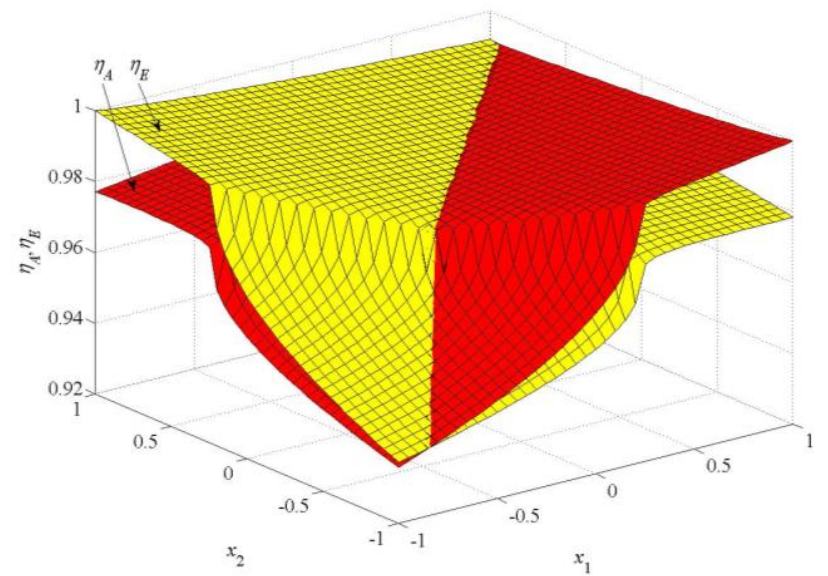

Fig. 2 The graphical representation of the (4) and (7) surfaces for $z_{1}=19, z_{2}=19$ and $\mu=0.05$

In order to obtain the graphical representation of the (4) and (6) surfaces the following input data were considered: $\mu_{A}=\mu_{E}=\mu=0.05$. The $x_{1}$ and $x_{2}$ values are in range $[-1,1]$ and the $z_{1}$ and $z_{2}$ values are fixed and the same for each surface representation. As seen in Fig. 2 the two surfaces intersect, so the equalization of the efficiencies, at the $\mathrm{A}$ and $\mathrm{E}$ points, have solutions. The curve from Fig. 3 was obtained using a Matlab code extracting the isoline of the intersection and then interpolating on surface (7). The results from these figures are however rough because:

- the solutions are obtained using Matlab facilities (surface intersection extraction and interpolation) instead of solving directly the $(4)=(7)$ equation;

- in order to obtain results for the surfaces as Matlab requires, no limitations on the obtained results were applied; these concern the values of the meshing angle and the $x_{1}$ and $x_{2}$ values to avoid tooth undercut and sharpening.

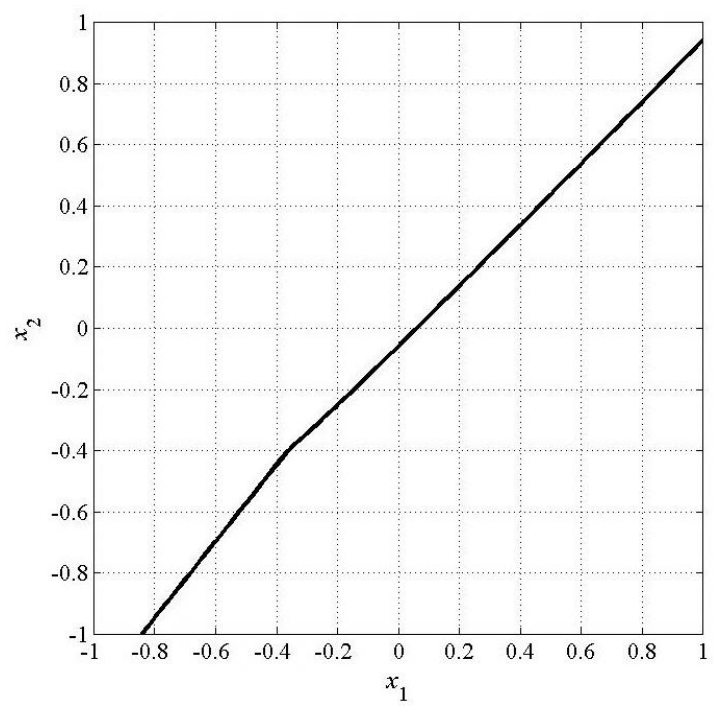

Fig. 3 The intersection curve of the (4) and (7) surfaces for $z_{1}=19, z_{2}=19$ and $\mu=0.05$

\section{Numerical results of the efficiency equalization}

To avoid the tooth undercut and sharpening the following limitations were applied to the obtained $x_{1}, x_{2}$ results [1], [3]:

- for tooth undercut:

$$
\begin{aligned}
& x_{1 \min }=\frac{17-z_{1}}{17} ; \\
& x_{2 \min }=\frac{17-z_{2}}{17} ;
\end{aligned}
$$

- for tooth sharpening by putting the following conditions:

$$
\begin{aligned}
& d_{a 1} \leq d_{v 1}-\frac{m}{6} \\
& d_{a 2} \leq d_{v 2}-\frac{m}{6}
\end{aligned}
$$

we obtain:

$$
\begin{aligned}
& x_{1 \max }=\frac{d_{v 1}}{2 m}-\frac{1}{12}-\frac{z_{1}}{2}-h_{a}^{*}+k, \\
& x_{2 \max }=\frac{d_{v 2}}{2 m}-\frac{1}{12}-\frac{z_{2}}{2}-h_{a}^{*}+k,
\end{aligned}
$$

with $d_{a 1}$ the outside circle diameter of wheel $1, d_{a 2}$ the outside circle diameter of wheel $2, d_{v 1}$ the sharp tip circle di- 
ameter of wheel $1, d_{v 2}$ the sharp tip circle diameter of wheel 2.

Equalization of the (4) and (7) relations give the following equation:

$\left[z_{1}\left(\mu_{A} \tan \left(\alpha_{w}\right)-1\right)-\mu_{A} z_{2}\left(\tan \left(\alpha_{A}-\tan \left(\alpha_{w}\right)\right)\right)\right] \times$

$\times\left[z_{2}\left(\mu_{E} \tan \left(\alpha_{E}\right)+1\right)-\mu_{E} z_{1}\left(\tan \left(\alpha_{E}\right)-\tan \left(\alpha_{w}\right)\right)\right]-$

$-z_{1} z_{2}\left(\mu_{A} \tan \left(\alpha_{A}\right)-1\right)\left(\mu_{E} \tan \left(\alpha_{E}\right)+1\right)=0$.

The Matlab code is based on the following equalization algorithm:

ha=1; a $0=\operatorname{deg} 2 \mathrm{rad}(20) ; \mathrm{miu}=0.05$;

$\mathrm{z} 1=19 ; \mathrm{z} 2=21$

$x 2 i n=-1.0 ; x 2$ fin=1.0;n=41;

step $=(x 2$ fin-x2in $) /(n-1)$;

plotcrt $1 \times 1=[]$;

plotcrt $1 \times 2=[]$;

for $i=1: n$

x2=x2in $+(i-1) *$ step;

o find alfa left and alfa right

을 the bisection method implemented

$\therefore$ in the bis function.

\% equalization equation to be solve is

\% passed as parameter to the bis

o solver function.

feq $=(23)$

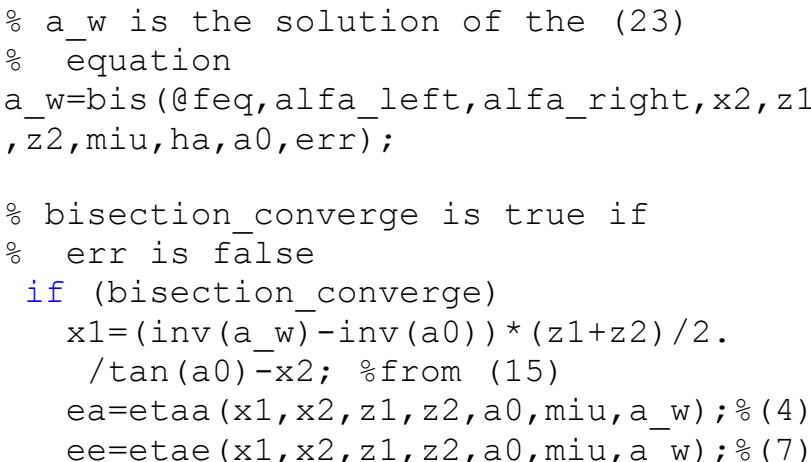

\% no limitation is true if

$\circ a \bar{w}$ in $\left[14^{0}, 32^{\circ}\right]$

$\circ \mathrm{x} 1$ in $\left[\mathrm{x}_{1 \min } . \mathrm{x}_{1 \max }\right]$ ofrom $(17)$, (18)

$\circ x 2$ in $\left[x_{2 \min }, x_{2 \max }\right]$ \%from (21), (22)

ㅇ abs (ea-ea) $<1 e-7$ recheck convergency

if (no_limitations)

print $i, x 1 \min (z 1), x 1$,

$\mathrm{x} 1$ max_v1 $(\mathrm{z} 1, \mathrm{z} 2, \mathrm{x} 1, \mathrm{x} 2)$,

$x 2^{-} \min (z 2), x 2$,

$\left.x^{-} \max v 1(\mathrm{z} 1, \mathrm{z} 2, \mathrm{x} 1, \mathrm{x} 2), \mathrm{a} w, \mathrm{ea}, \mathrm{ee}\right)$;

plotert $\overline{1} x 1=[$ plotcrt $1 \times 1 ; \bar{x} 1]$;

plotcrt $1 \times 2=[$ plotcrt $1 \times 2 ; x 2]$;

end if - no limitations

end if - bisection_converge

end $\%$ for

try

plot(plotcrt1x1, plotcrt1x2);

end

catch err

end

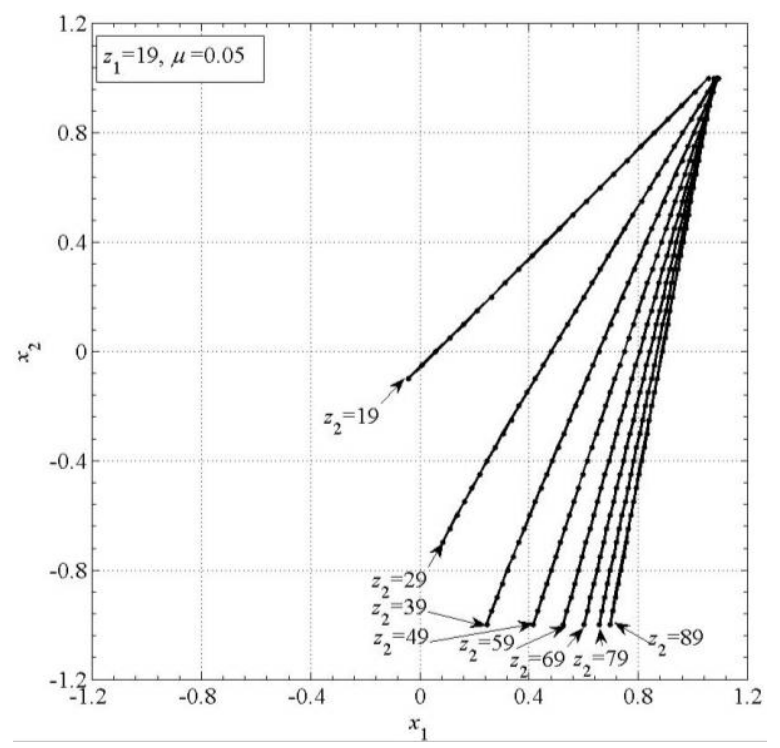

Fig. 4 The $x_{1}$ and $x_{2}$ plots for equalized efficiencies for $z_{1}=19, z_{2}=\{19,29,39, \ldots, 89\}$ and $\mu=0.05$

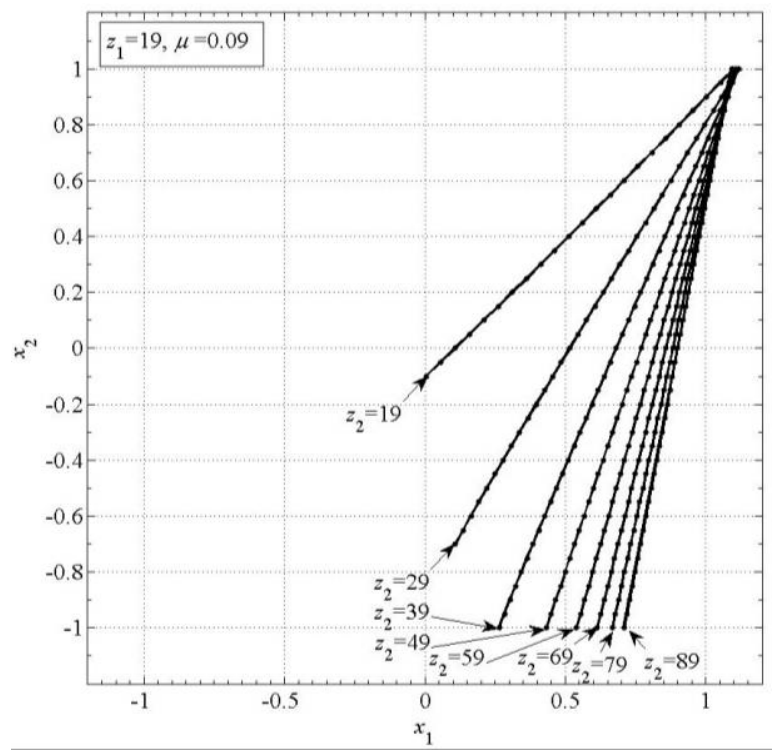

Fig. 5 The $x_{1}$ and $x_{2}$ plots for equalized efficiencies for $z_{1}=19, z_{2}=\{19,29,39, \ldots, 89\}$ and $\mu=0.09$

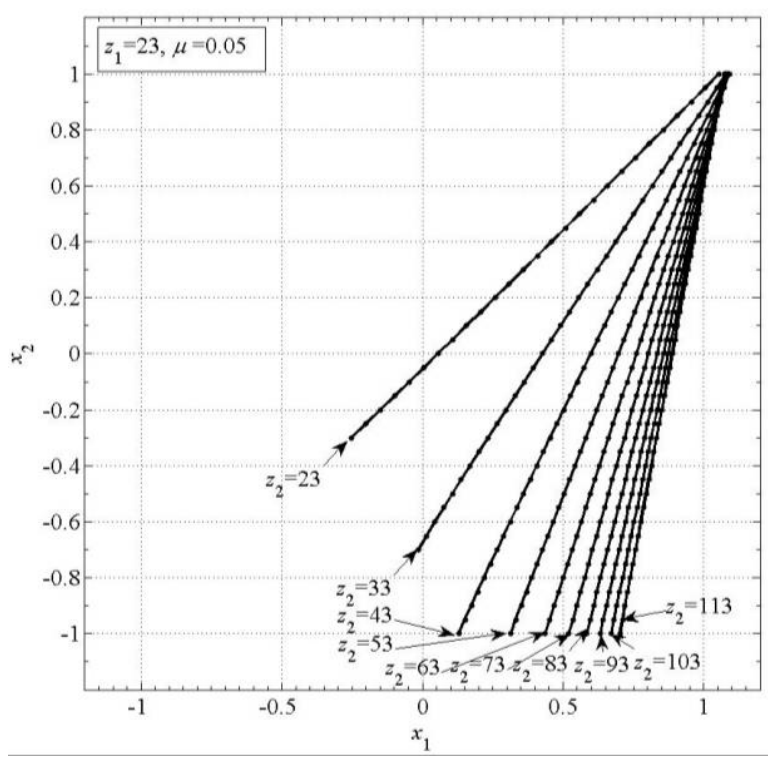

Fig. 6 The $x_{1}$ and $x_{2}$ plots for equalized efficiencies for $z_{1}=23, z_{2}=\{23,33,43, \ldots, 113\}$ and $\mu=0.05$ 


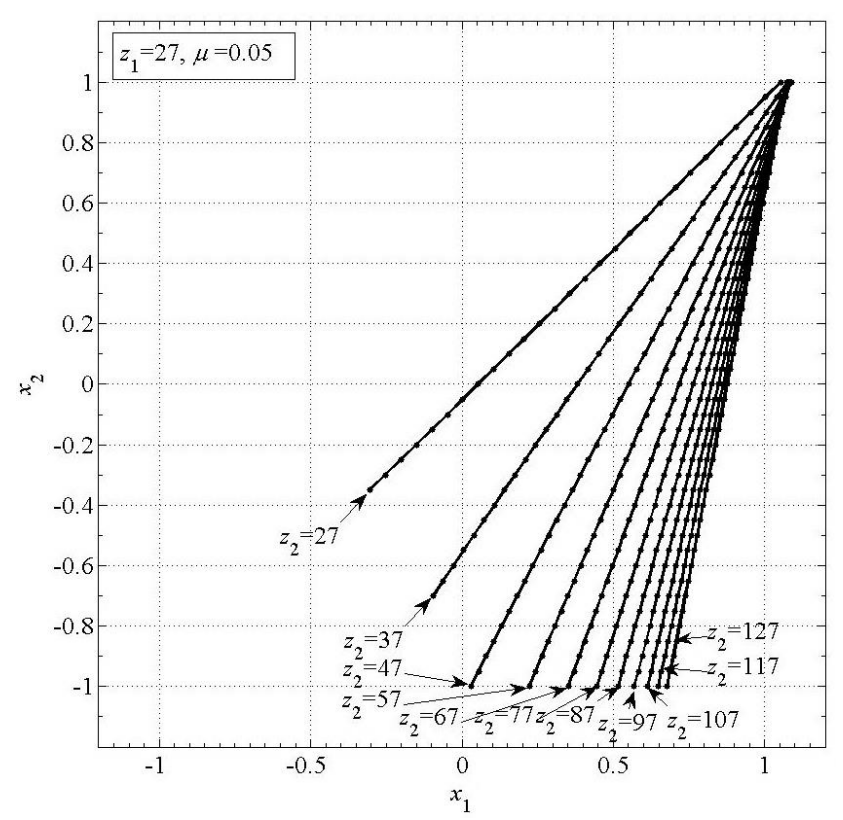

Fig. 7 The $x_{1}$ and $x_{2}$ plots for equalized efficiencies for $z_{1}=27, z_{2}=\{27,37,47, \ldots, 127\}$ and $\mu=0.05$

The results from Table 1 are showing the raw data produced by the Matlab code. The $[-1,1]$ domain is divid- ed into 41 points for $x_{2}$. Each solution is numbered from 1 to 41 in the $i$ column of Table 1. Missing lines are representing a lack of solution for the (23) equalization equation. Some of the lines from Table 1 are eliminated in Table 2 as $\alpha_{w}$ must be over $14^{\circ}$. Table 2 to Table 5 are condensed versions of valid solutions and together with the plots from Fig. 4 to Fig. 7 allow making the following conclusions:

- higher $\mu$ friction coefficient will increase the number of valid solutions, however because the variation of the friction coefficient is very limited its influence is low;

- higher number of teethe as high ratio of numbers of teeth favor the number of solutions;

- the equalized efficiencies are maximum for the highest values of the positive of the $x_{1}, x_{2}$ pair solution;

- $\quad$ high ratio of numbers of teeth favors a higher value of the equalized efficiencies.

The $x_{1}$ and $x_{2}$ values are chosen independently under normal circumstances. Imposing the equalization condition a relationship between these to independent variables is defined and only one, of the two, will remain independent. As seen in the algorithm, the $x_{2}$ values are given, while the $x_{1}$ values result from the equalization condition if (23) has valid solutions.

Table 1

Equalized efficiencies for $z_{1}=19, z_{2}=19$ and $\mu=0.05$ with limitations

\begin{tabular}{|c|c|c|c|c|c|c|c|c|c|}
\hline$i$ & $x_{I \min }$ & $x_{1}$ & $x_{1 \max }$ & $x_{2 \min }$ & $x_{2}$ & $x_{2 \max }$ & $\alpha_{w},{ }^{\circ}$ & $\eta_{A}$ & $\eta_{E}$ \\
\hline 14 & -0.11765 & -0.30546 & 0.42089 & -0.11765 & -0.35000 & 0.39399 & 10.92986 & 0.98440 & 0.98440 \\
\hline 15 & -0.11765 & -0.25140 & 0.38900 & -0.11765 & -0.30000 & 0.35982 & 13.38193 & 0.98521 & 0.98521 \\
\hline 16 & -0.11765 & -0.19844 & 0.38076 & -0.11765 & -0.25000 & 0.34998 & 15.12985 & 0.98576 & 0.98576 \\
\hline 17 & -0.11765 & -0.14612 & 0.38591 & -0.11765 & -0.20000 & 0.35393 & 16.52595 & 0.98620 & 0.98620 \\
\hline 18 & -0.11765 & -0.09426 & 0.40005 & -0.11765 & -0.15000 & 0.36715 & 17.70443 & 0.98657 & 0.98657 \\
\hline 19 & -0.11765 & -0.04274 & 0.42078 & -0.11765 & -0.10000 & 0.38716 & 18.73261 & 0.98690 & 0.98690 \\
\hline 20 & -0.11765 & 0.00851 & 0.44663 & -0.11765 & -0.05000 & 0.41246 & 19.64964 & 0.98720 & 0.98720 \\
\hline 21 & -0.11765 & 0.05953 & 0.47660 & -0.11765 & 0.00000 & 0.44200 & 20.48055 & 0.98749 & 0.98749 \\
\hline 22 & -0.11765 & 0.11036 & 0.50998 & -0.11765 & 0.05000 & 0.47508 & 21.24241 & 0.98776 & 0.98776 \\
\hline 23 & -0.11765 & 0.16102 & 0.54624 & -0.11765 & 0.10000 & 0.51113 & 21.94746 & 0.98802 & 0.98802 \\
\hline 24 & -0.11765 & 0.21153 & 0.58499 & -0.11765 & 0.15000 & 0.54975 & 22.60478 & 0.98827 & 0.98827 \\
\hline 25 & -0.11765 & 0.26190 & 0.62590 & -0.11765 & 0.20000 & 0.59061 & 23.22136 & 0.98851 & 0.98851 \\
\hline 26 & -0.11765 & 0.31216 & 0.66872 & -0.11765 & 0.25000 & 0.63344 & 23.80268 & 0.98875 & 0.98875 \\
\hline 27 & -0.1 & 0.36231 & 0.71324 & -0.1 & 0.30000 & 0.67803 & 24.35311 & 0.98898 & 0.98898 \\
\hline 28 & -0.11765 & 0.41235 & 0.75928 & -0.11765 & 0.35000 & 0.72419 & 24.87623 & 0.98921 & 0.98921 \\
\hline 29 & -0.11765 & 0.46231 & 0.80669 & -0.11765 & 0.40000 & 0.77179 & 25.37501 & 0.98944 & 0.98944 \\
\hline 30 & -0.11765 & 0.51218 & 0.85536 & -0.11765 & 0.45000 & 0.82067 & 25.85192 & 0.98966 & 0.98966 \\
\hline 31 & -0.11765 & 0.56197 & 0.90518 & -0.11765 & 0.50000 & 0.87074 & 26.30905 & 0.98988 & 0.98988 \\
\hline 32 & -0.11765 & 0.61168 & 0.95604 & -0.11765 & 0.55000 & 0.92190 & 26.74820 & 0.99010 & 0.99010 \\
\hline 33 & -0.11765 & 0.66133 & 1.00787 & -0.11765 & 0.60000 & 0.97406 & 27.17092 & 0.99031 & 0.99031 \\
\hline 34 & -0.11765 & 0.71090 & 1.06060 & -0.11765 & 0.65000 & 1.02715 & 27.57856 & 0.99053 & 0.99053 \\
\hline 35 & -0.11765 & 0.76042 & 1.11416 & -0.11765 & 0.70000 & 1.08110 & 27.97229 & 0.99074 & 0.99074 \\
\hline 36 & -0.11765 & 0.80988 & 1.16850 & -0.11765 & 0.75000 & 1.13586 & 28.35316 & 0.99095 & 0.99095 \\
\hline 37 & -0.11765 & 0.85928 & 1.22357 & -0.11765 & 0.80000 & 1.19137 & 28.72207 & 0.99116 & 0.99116 \\
\hline 38 & -0.11765 & 0.90863 & 1.27931 & -0.11765 & 0.85000 & 1.24758 & 29.07985 & 0.99137 & 0.99137 \\
\hline 39 & -0.11765 & 0.95792 & 1.33570 & -0.11765 & 0.90000 & 1.30445 & 29.42724 & 0.99158 & 0.99158 \\
\hline 40 & -0.11765 & 1.00717 & 1.39268 & -0.11765 & 0.95000 & 1.36195 & 29.76489 & 0.99179 & 0.99179 \\
\hline 41 & -0.11765 & 1.05637 & 1.45023 & -0.11765 & 1.00000 & 1.42003 & 30.09340 & 0.99199 & 0.99199 \\
\hline
\end{tabular}


Table 2

Equalized efficiencies for $z_{1}=19, z_{2}=19$ and $\mu=0.05$

\begin{tabular}{|c|c|c|c|c|}
\hline$i$ & $x_{1}$ & $x_{2}$ & $\alpha_{w}{ }^{\circ}$ & $\eta_{A}=\eta_{E}$ \\
\hline 19 & -0.04274 & -0.10000 & 18.73261 & 0.98690 \\
\hline 20 & 0.00851 & -0.05000 & 19.64964 & 0.98720 \\
\hline 21 & 0.05953 & 0.00000 & 20.48055 & 0.98749 \\
\hline 22 & 0.11036 & 0.05000 & 21.24241 & 0.98776 \\
\hline 23 & 0.16102 & 0.10000 & 21.94746 & 0.98802 \\
\hline 24 & 0.21153 & 0.15000 & 22.60478 & 0.98827 \\
\hline 25 & 0.26190 & 0.20000 & 23.22136 & 0.98851 \\
\hline 26 & 0.31216 & 0.25000 & 23.80268 & 0.98875 \\
\hline 27 & 0.36231 & 0.30000 & 24.35311 & 0.98898 \\
\hline 28 & 0.41235 & 0.35000 & 24.87623 & 0.98921 \\
\hline 29 & 0.46231 & 0.40000 & 25.37501 & 0.98944 \\
\hline 30 & 0.51218 & 0.45000 & 25.85192 & 0.98966 \\
\hline 31 & 0.56197 & 0.50000 & 26.30905 & 0.98988 \\
\hline 32 & 0.61168 & 0.55000 & 26.74820 & 0.99010 \\
\hline 33 & 0.66133 & 0.60000 & 27.17092 & 0.99031 \\
\hline 34 & 0.71090 & 0.65000 & 27.57856 & 0.99053 \\
\hline 35 & 0.76042 & 0.70000 & 27.97229 & 0.99074 \\
\hline 36 & 0.80988 & 0.75000 & 28.35316 & 0.99095 \\
\hline 37 & 0.85928 & 0.80000 & 28.72207 & 0.99116 \\
\hline 38 & 0.90863 & 0.85000 & 29.07985 & 0.99137 \\
\hline 39 & 0.95792 & 0.90000 & 29.42724 & 0.99158 \\
\hline 40 & 1.00717 & 0.95000 & 29.76489 & 0.99179 \\
\hline 41 & 1.05637 & 1.00000 & 30.09340 & 0.99199 \\
\hline
\end{tabular}

Table 3

Equalized efficiencies for $z_{1}=19, z_{2}=89$ and $\mu=0.05$

\begin{tabular}{|c|c|c|c|c|}
\hline$i$ & $x_{1}$ & $x_{2}$ & $\alpha_{w}{ }^{\circ}$ & $\eta_{A}=\eta_{E}$ \\
\hline 1 & 0.69735 & -1.00000 & 19.07191 & 0.99578 \\
\hline 2 & 0.71657 & -0.90000 & 19.44898 & 0.99581 \\
\hline 3 & 0.73575 & -0.80000 & 19.81077 & 0.99585 \\
\hline 4 & 0.75490 & -0.70000 & 20.15867 & 0.99588 \\
\hline 5 & 0.77402 & -0.60000 & 20.49389 & 0.99592 \\
\hline 6 & 0.79311 & -0.50000 & 20.81751 & 0.99596 \\
\hline 7 & 0.81219 & -0.40000 & 21.13043 & 0.99599 \\
\hline 8 & 0.83125 & -0.30000 & 21.43347 & 0.99603 \\
\hline 9 & 0.85030 & -0.20000 & 21.72733 & 0.99608 \\
\hline 10 & 0.86934 & -0.10000 & 22.01267 & 0.99612 \\
\hline 11 & 0.88838 & 0.00000 & 22.29003 & 0.99616 \\
\hline 12 & 0.90741 & 0.10000 & 22.55994 & 0.99621 \\
\hline 13 & 0.92644 & 0.20000 & 22.82286 & 0.99625 \\
\hline 14 & 0.94547 & 0.30000 & 23.07920 & 0.99630 \\
\hline 15 & 0.96450 & 0.40000 & 23.32933 & 0.99634 \\
\hline 16 & 0.98354 & 0.50000 & 23.57361 & 0.99639 \\
\hline 17 & 1.00259 & 0.60000 & 23.81235 & 0.99644 \\
\hline 18 & 1.02164 & 0.70000 & 24.04583 & 0.99649 \\
\hline 19 & 1.04070 & 0.80000 & 24.27433 & 0.99654 \\
\hline 20 & 1.05978 & 0.90000 & 24.49808 & 0.99659 \\
\hline 21 & 1.07886 & 1.00000 & 24.71732 & 0.99664 \\
\hline
\end{tabular}

Table 4

Equalized efficiencies for $z_{1}=27, z_{2}=27$ and $\mu=0.05$

\begin{tabular}{|c|c|c|c|c|}
\hline$i$ & $x_{1}$ & $x_{2}$ & $\alpha_{w},{ }^{\circ}$ & $\eta_{A}=\eta_{E}$ \\
\hline 14 & -0.30538 & -0.35000 & 14.93574 & 0.98926 \\
\hline 15 & 0.25338 & -0.30000 & 15.96645 & 0.98951 \\
\hline 16 & 0.20167 & -0.25000 & 16.87081 & 0.98973 \\
\hline 17 & -0.15021 & -0.20000 & 17.68081 & 0.98994 \\
\hline 18 & -0.09896 & -0.15000 & 18.41716 & 0.99013 \\
\hline 19 & -0.04788 & -0.10000 & 19.09416 & 0.99031 \\
\hline 20 & 0.00305 & -0.05000 & 19.72211 & 0.99049 \\
\hline 21 & 0.05385 & 0.00000 & 20.30874 & 0.99066 \\
\hline 22 & 0.10452 & 0.05000 & 20.85997 & 0.99082 \\
\hline 23 & 0.15508 & 0.10000 & 21.38049 & 0.99098 \\
\hline 24 & 0.20555 & 0.15000 & 21.87408 & 0.99114 \\
\hline 25 & 0.25592 & 0.20000 & 22.34380 & 0.99129 \\
\hline 26 & 0.30620 & 0.25000 & 22.79221 & 0.99145 \\
\hline 27 & 0.35641 & 0.30000 & 23.22146 & 0.99160 \\
\hline 28 & 0.40654 & 0.35000 & 23.63335 & 0.99175 \\
\hline 29 & 0.45660 & 0.40000 & 24.02946 & 0.99189 \\
\hline 30 & 0.50660 & 0.45000 & 24.41111 & 0.99204 \\
\hline 31 & 0.55653 & 0.50000 & 24.77949 & 0.99219 \\
\hline 32 & 0.60641 & 0.55000 & 25.13561 & 0.99233 \\
\hline 33 & 0.65623 & 0.60000 & 25.48040 & 0.99248 \\
\hline 34 & 0.70599 & 0.65000 & 25.81464 & 0.99262 \\
\hline 35 & 0.75571 & 0.70000 & 26.13906 & 0.99276 \\
\hline 36 & 0.80538 & 0.75000 & 26.45429 & 0.99291 \\
\hline 37 & 0.85500 & 0.80000 & 26.76093 & 0.99305 \\
\hline 38 & 0.90458 & 0.85000 & 27.05947 & 0.99319 \\
\hline 39 & 0.95411 & 0.90000 & 27.35041 & 0.99333 \\
\hline 40 & 1.00360 & 0.95000 & 27.63416 & 0.99347 \\
\hline 41 & 1.05306 & 1.00000 & 27.91113 & 0.99361 \\
\hline
\end{tabular}

Table 5

Equalized efficiencies for $z_{1}=27, z_{2}=97$ and $\mu=0.05$

\begin{tabular}{|l|c|c|c|c|}
\hline \multicolumn{1}{|c|}{$i$} & $x_{1}$ & $x_{2}$ & $\alpha_{w}{ }^{\circ}$ & $\eta_{A}=\eta_{E}$ \\
\hline 1 & 0.56942 & -1.00000 & 18.83493 & 0.99598 \\
\hline 2 & 0.58231 & -0.95000 & 19.01489 & 0.99599 \\
\hline 3 & 0.59519 & -0.90000 & 19.19121 & 0.99601 \\
\hline 4 & 0.60806 & -0.85000 & 19.36407 & 0.99603 \\
\hline 5 & 0.62092 & -0.80000 & 19.53362 & 0.99604 \\
\hline 6 & 0.63377 & -0.75000 & 19.70003 & 0.99606 \\
\hline 7 & 0.64660 & -0.70000 & 19.86342 & 0.99608 \\
\hline 8 & 0.65943 & -0.65000 & 20.02392 & 0.99609 \\
\hline 9 & 0.67225 & -0.60000 & 20.18165 & 0.99611 \\
\hline 10 & 0.68507 & -0.55000 & 20.33673 & 0.99613 \\
\hline 11 & 0.69787 & -0.50000 & 20.48926 & 0.99615 \\
\hline 12 & 0.71067 & -0.45000 & 20.63933 & 0.99617 \\
\hline 13 & 0.72346 & -0.40000 & 20.78705 & 0.99619 \\
\hline 14 & 0.73625 & -0.35000 & 20.93249 & 0.99621 \\
\hline 15 & 0.74904 & -0.30000 & 21.07574 & 0.99623 \\
\hline 16 & 0.76182 & -0.25000 & 21.21688 & 0.99625 \\
\hline 17 & 0.77459 & -0.20000 & 21.35598 & 0.99627 \\
\hline
\end{tabular}




\begin{tabular}{|c|c|c|c|c|}
\hline$i$ & $x_{1}$ & $x_{2}$ & $\alpha_{w},{ }^{\circ}$ & $\eta_{A}=\eta_{E}$ \\
\hline 18 & 0.78737 & -0.15000 & 21.49311 & 0.99629 \\
\hline 19 & 0.80014 & -0.10000 & 21.62834 & 0.99631 \\
\hline 20 & 0.81290 & -0.05000 & 21.76172 & 0.99633 \\
\hline 21 & 0.82567 & 0.00000 & 21.89332 & 0.99635 \\
\hline 22 & 0.83843 & 0.05000 & 22.02318 & 0.99637 \\
\hline 23 & 0.85120 & 0.10000 & 22.15138 & 0.99640 \\
\hline 24 & 0.86396 & 0.15000 & 22.27795 & 0.99642 \\
\hline 25 & 0.87672 & 0.20000 & 22.40295 & 0.99644 \\
\hline 26 & 0.88948 & 0.25000 & 22.52641 & 0.99646 \\
\hline 27 & 0.90224 & 0.30000 & 22.64839 & 0.99649 \\
\hline 28 & 0.91500 & 0.35000 & 22.76893 & 0.99651 \\
\hline 29 & 0.92776 & 0.40000 & 22.88806 & 0.99653 \\
\hline 30 & 0.94052 & 0.45000 & 23.00583 & 0.99655 \\
\hline 31 & 0.95328 & 0.50000 & 23.12226 & 0.99658 \\
\hline 32 & 0.96605 & 0.55000 & 23.23741 & 0.99660 \\
\hline 33 & 0.97881 & 0.60000 & 23.35129 & 0.99663 \\
\hline 34 & 0.99158 & 0.65000 & 23.46395 & 0.99665 \\
\hline 35 & 1.00435 & 0.70000 & 23.57541 & 0.99667 \\
\hline 36 & 1.01711 & 0.75000 & 23.68570 & 0.99670 \\
\hline 37 & 1.02989 & 0.80000 & 23.79485 & 0.99672 \\
\hline 38 & 1.04266 & 0.85000 & 23.90289 & 0.99675 \\
\hline 39 & 1.05544 & 0.90000 & 24.00986 & 0.99677 \\
\hline 40 & 1.06822 & 0.95000 & 24.11575 & 0.99680 \\
\hline 41 & 1.08100 & 1.00000 & 24.22062 & 0.99682 \\
\hline
\end{tabular}

77(3): 53-57.

5. Niemann, G.; Winter, H. 1983. Maschine parts. Vol. III, -Berlin, Springer-Verlag: 1-19 (in German).

6. DUBBEL 1997. Handbook of Machine Elements, Edition 19, Springer Verlag, G 137-141 (in German).

7. Niemann, G.; Winter, H. 2003. Maschinenelemente, Band 2, Berlin: Springer-Verlag. 376p. (in German).

\section{T. A. Antal}

\section{ADDENDUM MODIFICATION OF SPUR GEARS WITH EQUALISED EFFICIENCY AT THE POINTS WHERE THE MESHING STARS AND}

S u m m a r y

The paper gives a new method for obtaining the geometrical dimensions for involute spur gears based on the equalization conditions of the efficiencies, at the A and E points, where the meshing begins and ends. Numerical results and plots for the $x_{1}$ and $x_{2}$ addendum modifications are obtained, using the Matlab programming environment. The solutions are obtained for different pairs of teeth numbers, when the equalization condition stands, together with the limitations given by the teeth undercut, sharpening and operating pressure angle.

Keywords: spur gears, addendum modification, efficiency equalization.

Received June 11, 2015

Accepted September17, 2015

The values of the specific addendum modifications $x_{1}$ and $x_{2}$ can be determined by this equalization procedure. A balanced efficiency will lead to balanced efficiency loss. As the efficiency loss is connected to sliding and rolling frictional losses the equalization will lead to a uniform wearing of teeth flanks at the points where the meshing ends and begins during load transmission. The algorithm allows finding a better balanced efficiency where the power losses are reduced. Reduced power losses lead to better operating conditions (lower operating temperature, noise and wear).

\section{References}

1. Baglioni, S.; Cianetti, F.; Landi, L. 2012. Influence of the addendum modification on spur gear efficiency, Mechanism and Machine Theory 49: 216-233. http://dx.doi.org/10.1016/j.mechmachtheory.2011.10.0 07.

2. Li, X.; Jiang, S.; Zeng, Q. 2013. Optimal selection of addendum modification coefficients of involute cylindrical gears, Journal of Computers 8(8): 2156-2161.

3. Antal, A.T. 2014. Addendum modification of cylindrical spur gears with equalized powers lost by friction between the teeth flanks at the points where the meshing stars and ends, Acta Technica Napocensis Series: Applied Mathematics, Mechanics and Engineering 75(3): 329-334.

4. Antal, A.T. 2009. A new algorithm for helical gear design with addendum modification, Mechanika 